\title{
Nucleon thermal width owing to pion-baryon loops and its contributions to shear viscosity
}

\author{
Sabyasachi Ghosh \\ Instituto de Fisica Teorica, Universidade Estadual Paulista, Rua Dr. Bento Teobaldo Ferraz, 271, 01140-070 Sao Paulo, SP, Brazil
}

(Received 30 December 2013; revised manuscript received 23 June 2014; published 7 August 2014)

\begin{abstract}
In real-time thermal field theory, the standard expression of shear viscosity for nucleonic constituents is derived from the two-point function of nucleonic viscous stress tensors at finite temperature and density. The finite thermal width or Landau damping is traditionally included in the nucleon propagators. This thermal width is calculated from the in-medium self-energy of nucleons for different possible pion-baryon loops. The dynamical part of nucleon-pion-baryon interactions are accounted for by the effective Lagrangian densities of standard hadronic model. The shear viscosity to entropy density ratio of the nucleonic component decreases with the temperature and increases with the nucleon chemical potential. However, adding the contribution of the pionic component, the total viscosity to entropy density ratio also reduces with the nucleon chemical potential when the mixing effect between pion and nucleon components in the mixed gas is considered. Within the hadronic domain, the viscosity to entropy density ratio of the nuclear matter gradually reduces as temperature and nucleon chemical potential increase and therefore the nuclear matter is approaching the (nearly) perfect-fluid state.
\end{abstract}

DOI: 10.1103/PhysRevC.90.025202

PACS number(s): 24.85.+p, 13.75.Gx, 14.20.Dh, 25.75.-q

\section{INTRODUCTION}

Recent hydrodynamical studies [1,2], as well as some transport studies $[3,4]$, have indicated about a (nearly) ideal fluid nature of nuclear matter, which may be produced in the experiments of heavy-ion collisions (HICs) such as at the Relativistic Heavy Ion Collider (RHIC) at BNL. The hydrodynamical calculations became very successful in explaining the elliptical flow parameter $v_{2}$ from RHIC data [5-7] only when they assumed a very small ratio of shear viscosity to entropy density $(\eta / s)$ for the expanding nuclear matter. When some recent studies [8-11] (see also Ref. [12]) showed that $\eta / s$ may reach a minimum in the vicinity of a phase transition, some special attention was drawn to the smallness of this minimum with respect to its lower bound $\left(\eta / s=\frac{1}{4 \pi}\right)$, commonly known as the KSS bound [13]. In this context, the temperature $(T)$ dependence of $\eta / s$ is taken into account in some recent hydrodynamical calculations [14-17] instead of its constant value during the entire evolution. Niemi et al. [14] have interestingly observed that the $v_{2}\left(p_{T}\right)$ of RHIC data is highly sensitive to the temperature-dependent $\eta / s$ in hadronic matter and almost independent of the viscosity in the quark-gluon-plasma (QGP) phase. This work gives an additional boost to the microscopic calculations of $\eta / s$ of the hadronic matter in the recent years [18-34], although historically these investigations are slightly old [35-39].

Except for a few studies [24,30-32], most of the microscopic calculations are done at zero baryon or nucleon chemical potential $\left(\mu_{N}=0\right)$. Along with the $T$ dependence of $\eta$ or $\eta / s$, their dependence on the baryon chemical potential should also be understood in view of the future experiments such as at the GSI Facility for Antiproton and Ion Research (FAIR). In the work of Itakura et al. [24] and Denicol et al. [31], we notice that $\eta / s$ is reduced at finite baryon chemical potential, whereas Gorenstein et al. [30] observed an increasing nature of $\eta / s$ with $\mu_{N}$. Itakura $e t$ al. obtained $\eta$ by solving the relativistic quantum Boltzmann equation, where phenomenological amplitudes of hadrons are used in the collision terms. Denicol et al. calculated $\eta$ at finite $T$ and $\mu_{N}$ by applying Chapman-Enskog theory in a hadron resonance gas (HRG) model, whereas Gorenstein et al. took a simplified ansatz of $\eta$ to estimate $\eta / s$ in the van der Waals excluded volume HRG model. Similar to the ansatz of $\eta(T)$ taken by Gorenstein et al., $\eta$ itself increases with increasing temperature in Ref. [24], but their $\eta / s$ exhibit the completely opposite nature of $T$ dependence. Therefore, the behavior of the $\eta / s$ may largely be influenced by the $T$ dependence of the entropy density $s$.

Motivated by these delicate issues of shear viscosity at finite $\mu_{N}$, the present paper is concentrated on the matter with nucleon degrees of freedom at finite $T$ and $\mu_{N}$. The nucleons in the medium can slightly become off-equilibrium because of their thermal width or Landau damping, which can originate from the nucleon thermal fluctuations into different baryons and pions. The inverse of nucleon thermal width measures the relaxation time of nucleons in the matter, from which one can estimate its corresponding shear viscosity contribution.

In the next section, the one-loop expression of $\eta$ for nucleon degrees of freedom is derived from the Kubo relation, where a finite thermal width is traditionally included in the nucleon propagators. This standard expression of $\eta$ can also be deduced from the relaxation-time approximation of kinematic theory. In real-time thermal field theory, the nucleon thermal width from the different pion-baryon loops is calculated in Sec. III, where their interactions are determined from the effective hadronic model. In Sec. IV, the numerical results are discussed followed by summary and conclusions in Sec. V.

\section{KUBO RELATION FOR SHEAR VISCOSITY OF NUCLEAR MATTER}

From the simple derivation of the Kubo formula [40,41], let us start with the expression of shear viscosity for nucleonic constituents in momentum space [26,42],

$$
\eta_{N}=\frac{1}{20} \lim _{q_{0}, \vec{q} \rightarrow 0} \frac{A_{\eta}\left(q_{0}, \vec{q}\right)}{q_{0}}
$$


where

$$
A_{\eta}\left(q_{0}, \vec{q}\right)=\int d^{4} x e^{i q \cdot x}\left\langle\left[\pi_{\mu \nu}(x), \pi^{\mu \nu}(0)\right]\right\rangle_{\beta}
$$

is the spectral representation of the two-point function for the nucleonic viscous-stress tensor $\pi^{\mu \nu}$ and

$$
\langle\hat{O}\rangle_{\beta}=\operatorname{Tr} \frac{e^{-\beta H} \hat{O}}{Z}, \quad \text { with } \quad Z=\operatorname{Tr} e^{-\beta H}
$$

denotes the thermodynamical ensemble average. The energymomentum tensor of free nucleons is

$$
\begin{aligned}
T_{\rho \sigma} & =-g_{\rho \sigma} \mathcal{L}+\frac{\partial \mathcal{L}}{\partial\left(\partial^{\rho} \psi\right)} \partial_{\sigma} \psi \\
& =-g_{\rho \sigma} \mathcal{L}+i \bar{\psi} \gamma_{\rho} \partial_{\sigma} \psi,
\end{aligned}
$$

and hence the viscous stress tensor will be

$$
\begin{aligned}
\pi_{\mu \nu} & =t_{\mu \nu}^{\rho \sigma} T_{\rho \sigma} \\
& =t_{\mu \nu}^{\rho \sigma} i \bar{\psi} \gamma_{\rho} \partial_{\sigma} \psi\left(\text { since } t_{\mu \nu}^{\rho \sigma} g_{\rho \sigma}=0\right),
\end{aligned}
$$

where

$$
t_{\mu \nu}^{\rho \sigma}=\Delta_{\mu}^{\rho} \Delta_{v}^{\sigma}-\frac{1}{3} \Delta_{\mu v} \Delta^{\rho \sigma}, \quad \Delta^{\mu v}=g^{\mu v}-u^{\mu} u^{v} .
$$

In real-time formalism of thermal field theory, the ensemble average of any two-point function always becomes a $2 \times 2$ matrix structure. Hence, for the viscous-stress tensor, the matrix structure of the two-point function becomes

$$
\Pi_{a b}(q)=i \int d^{4} x e^{i q x}\left\langle T_{c} \pi_{\mu \nu}(x) \pi^{\mu v}(0)\right\rangle_{\beta}^{a b},
$$

where the superscripts $a, b(a, b=1,2)$ denote the thermal indices of the matrix and $T_{c}$ denotes time ordering with respect to a symmetrical contour $[43,44]$ in the complex time plane.

The matrix can be diagonalized in terms of a single analytic function, which can also be related with the retarded two-point function of the viscous-stress tensor. The retarded function $\Pi^{R}(q)$, diagonal element $\bar{\Pi}(q)$, and the spectral function $A_{\eta}(q)$ are simply related to any one of the components of $\Pi_{a b}(q)$. Their relations with the 11 component is given below

$$
\begin{aligned}
A_{\eta}(q) & =2 \operatorname{Im} \Pi^{R}(q)=2 \epsilon\left(q_{0}\right) \operatorname{Im} \bar{\Pi}(q) \\
& =2 \tanh \left(\frac{\beta q_{0}}{2}\right) \operatorname{Im} \Pi_{11}(q) .
\end{aligned}
$$

Hence, Eq. (1) can broadly be redefined as

$$
\begin{aligned}
\eta_{N} & =\frac{1}{10} \lim _{q_{0}, \vec{q} \rightarrow 0} \frac{\operatorname{Im} \Pi^{R}\left(q_{0}, \vec{q}\right)}{q_{0}}=\frac{1}{10} \lim _{q_{0}, \vec{q} \rightarrow 0} \frac{\epsilon\left(q_{0}\right) \operatorname{Im} \bar{\Pi}\left(q_{0}, \vec{q}\right)}{q_{0}} \\
& =\frac{1}{10} \lim _{q_{0}, \vec{q} \rightarrow 0} \frac{\tanh \left(\beta q_{0} / 2\right) \operatorname{Im} \Pi^{11}\left(q_{0}, \vec{q}\right)}{q_{0}} .
\end{aligned}
$$

Using Eq. (5) in the 11 component of Eq. (7) and then applying Wick's contraction technique, we have

$$
\begin{aligned}
& \Pi_{11}(q) \\
& =t_{\alpha \beta}^{\rho \sigma} t_{\mu \nu}^{\alpha \beta} i \int d^{4} x e^{i q x}\left\langle T \bar{\psi}(\underbrace{(x) \gamma_{\rho} \partial_{\sigma} \psi \overbrace{(x) \bar{\psi}}(0) \gamma^{\mu} \partial^{\nu} \psi}(0)\rangle_{\beta}\right. \\
& \quad=i \int \frac{d^{4} k}{(2 \pi)^{4}} N(q, k) D_{11}(k) D_{11}(p=q+k)
\end{aligned}
$$

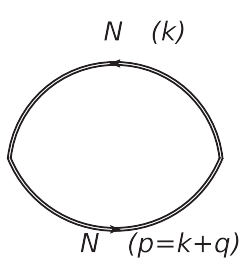

(a)

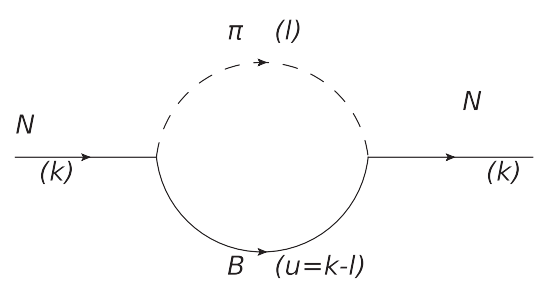

(b)
FIG. 1. Diagrammatic representation of $N N$ loop is shown in panel (a), where the double lines stand for effective $N$ propagators, which contain their thermal widths $\Gamma$. The diagrammatic representation of nucleon self-energy for the $\pi B$ loop is shown in panel (b) from where $\Gamma$ can be determined.

where

$$
\begin{aligned}
N(q, k)= & -I_{N} t_{\mu \nu}^{\rho \sigma} \operatorname{Tr}\left[\gamma^{\mu}(q+k)^{\nu}\left(\not q+\not k+m_{N}\right)\right. \\
& \left.\times \gamma_{\rho} k_{\sigma}\left(\not k+m_{N}\right)\right] .
\end{aligned}
$$

This self-energy function $\Pi_{11}(q)$ for the $N N$ loop can be represented diagrammatically by Fig. 1(a). In the comoving frame, i.e., for $u=(1, \overrightarrow{0}), N(q, k)$ becomes

$$
\begin{aligned}
N(q, k)= & -I_{N}\left[\frac{32}{3}\left\{k_{0}\left(q_{0}+k_{0}\right)\right\}\{\vec{k} \cdot(\vec{q}+\vec{k})\}\right. \\
& \left.-4\left[\{\vec{k} \cdot(\vec{q}+\vec{k})\}^{2}+\frac{\vec{k}^{2}(\vec{q}+\vec{k})^{2}}{3}\right]\right] .
\end{aligned}
$$

In the above equations, $I_{N}=2$ is the isospin degeneracy of the nucleon.

In Eq. (10), $D^{11}$ is the scalar part of the 11 component of the nucleon propagator at finite temperature and density. Its form is

$$
\begin{aligned}
D^{11}(k)= & \frac{-1}{k^{2}-m_{N}^{2}+i \eta}-2 \pi i F_{k}\left(k_{0}\right) \delta\left(k^{2}-m_{N}^{2}\right) \\
= & -\frac{1}{2 \omega_{k}^{N}}\left(\frac{1-n_{k}^{+}}{k_{0}-\omega_{k}^{N}+i \eta}+\frac{n_{k}^{+}}{k_{0}-\omega_{k}^{N}-i \eta}\right. \\
& \left.-\frac{1-n_{k}^{-}}{k_{0}+\omega_{k}^{N}-i \eta}-\frac{n_{k}^{-}}{k_{0}+\omega_{k}^{N}+i \eta}\right),
\end{aligned}
$$

with $F_{k}\left(k_{0}\right)=n_{k}^{+} \theta\left(k_{0}\right)+n_{k}^{-} \theta\left(-k_{0}\right)$ and where $n_{k}^{ \pm}\left(\omega_{k}^{N}\right)=$ $1 /\left\{e^{\beta\left(\omega_{k}^{N} \mp \mu_{N}\right)}+1\right\}$ is Fermi-Dirac distribution function for energy $\omega_{k}^{N}=\left(\vec{k}^{2}+m_{N}^{2}\right)^{1 / 2}$. Here the \pm signs in the superscript of $n_{k}$ stand for nucleon and antinucleon, respectively. Among the four terms in Eq. (13), the first and the second terms are associated with the nucleon propagation above the Fermi sea and the propagation of its hole in the Fermi sea, respectively, while the third and fourth terms represent the corresponding situations for the antinucleon. The full relativistic nucleon propagator, thus, treats the particle and antiparticle on an equal footing and all possible singularities (nucleon, hole of the nucleon, antinucleon, and hole of the antinucleon) are automatically included. 
After doing the $k_{0}$ integration of Eq. (10) and then using it in Eq. (9), we have

$$
\begin{aligned}
\eta_{N}= & \frac{1}{10} \lim _{q_{0}, \vec{q} \rightarrow 0} \int \frac{d^{3} k}{(2 \pi)^{3}} \frac{(-\pi N)}{4 \omega_{k}^{N} \omega_{p}^{N}} \\
& \times\left[\frac{\left\{-n_{k}^{-}\left(\omega_{k}^{N}\right)+n_{p}^{-}\left(-q_{0}+\omega_{k}^{N}\right)\right\}}{q_{0}} \delta\left(q_{0}-\omega_{k}^{N}+\omega_{p}^{N}\right)\right. \\
& \left.+\frac{\left\{n_{k}^{+}\left(\omega_{k}^{N}\right)-n_{p}^{+}\left(q_{0}+\omega_{k}^{N}\right)\right\}}{q_{0}} \delta\left(q_{0}+\omega_{k}^{N}-\omega_{p}^{N}\right)+\cdots\right],
\end{aligned}
$$

where $\quad N=N\left(k_{0}= \pm \omega_{k}^{N}, \vec{k}, q\right) \quad$ and $\quad \omega_{p}^{N}=\left[(\vec{q}+\vec{k})^{2}+\right.$ $\left.m_{N}^{2}\right]^{1 / 2}$.

The two $\delta$ functions will be responsible for generating the Landau cuts $\left(-\vec{q}<q_{0}<\vec{q}\right)$, where the $\operatorname{Im} \Pi^{R}(q)$ will be nonzero. However, there will be two more $\delta$ functions (not written explicitly), which are not important for the limiting point $q_{0}, \vec{q} \rightarrow 0$ since they will generate unitary cuts $[-\infty<$ $q_{0}<-\left(\vec{q}^{2}+4 m_{N}^{2}\right)^{1 / 2}$ and $\left.\left(\vec{q}^{2}+4 m_{N}^{2}\right)^{1 / 2}<q_{0}<\infty\right]$.

By using the identity

$$
-\pi \delta(x)=\operatorname{Im}\left[\lim _{\Gamma_{N} \rightarrow 0} \frac{1}{x+i \Gamma_{N}}\right]
$$

in Eq. (14), we have

$$
\begin{aligned}
\eta_{N}= & \frac{1}{10} \lim _{q_{0}, \vec{q} \rightarrow 0} \operatorname{Im}\left[\int \frac{d^{3} k}{(2 \pi)^{3}} \frac{N}{4 \omega_{k}^{N} \omega_{p}^{N}}\right. \\
& \times \lim _{\Gamma_{N} \rightarrow 0}\left\{\frac{\left\{-n_{k}^{-}\left(\omega_{k}^{N}\right)+n_{p}^{-}\left(-q_{0}+\omega_{k}^{N}\right)\right\} / q_{0}}{\left(q_{0}-\omega_{k}^{N}+\omega_{p}^{N}\right)+i \Gamma_{N}}\right. \\
& \left.\left.+\frac{\left\{n_{k}^{+}\left(\omega_{k}^{N}\right)-n_{p}^{+}\left(q_{0}+\omega_{k}^{N}\right)\right\} / q_{0}}{\left(q_{0}+\omega_{k}^{N}-\omega_{p}^{N}\right)+i \Gamma_{N}}\right\}\right] .
\end{aligned}
$$

We continue our further calculation for finite values of $\Gamma_{N}$ to get a nondivergent contribution of $\eta_{N}$. Including the thermal width $\Gamma_{N}$ for constituent particles (here nucleons) of the medium is a very-well-established technique $[26,27,45]$ in the Kubo approach to remove the divergence of $\eta_{N}$ as well as to incorporate the interaction scenario, which is very essential for a dissipative system. The interaction scenario is coming into the picture by transforming the delta functions to the spectral functions with finite thermal width. The thermal width (or collision rate) $\Gamma_{N}$ of the constituent particles reciprocally measures the shear viscosity coefficient, which is approximately equivalent to the quasiparticle description.

In the limiting case of $q_{0}, \vec{q} \rightarrow 0$, we get $\omega_{p}^{N} \rightarrow \omega_{k}^{N}$ and therefore Eq. (16) is transformed to

$$
\eta_{N}=\frac{1}{10} \int \frac{d^{3} k}{(2 \pi)^{3}} \frac{\left(-N_{0}\right)}{4 \omega_{k}^{N^{2}} \Gamma_{N}}\left[I_{2}+I_{3}\right]
$$

where

$$
N_{0}=\lim _{q_{0}, \vec{q} \rightarrow 0} N\left(k_{0}= \pm \omega_{k}^{N}, \vec{k}, q\right)
$$

and

$$
I_{2,3}=\lim _{q_{0} \rightarrow 0} \frac{\left\{\mp n_{k}^{\mp}\left(\omega_{k}^{N}\right) \pm n_{p}^{\mp}\left(\mp q_{0}+\omega_{k}^{N}\right)\right\}}{q_{0}} .
$$

In the above Eq. (19), one can notice that the limiting value of $I_{2,3}$ is of the $0 / 0$ form. Therefore, we can apply l'Hôspital's rule, i.e.,

$$
\begin{aligned}
I_{2,3} & =\lim _{q_{0} \rightarrow 0} \frac{\frac{d}{d q_{0}}\left\{\mp n_{k}^{\mp}\left(\omega_{k}^{N}\right) \pm n_{p}^{\mp}\left(\mp q_{0}+\omega_{k}^{N}\right)\right\}}{\frac{d}{d q_{0}}\left\{q_{0}\right\}} \\
& =\beta\left[n_{k}^{\mp}\left(1-n_{k}^{\mp}\right)\right],
\end{aligned}
$$

since

$$
\begin{aligned}
\frac{d}{d q_{0}}\left\{ \pm n_{p}^{\mp} \leqslant\left(\omega_{q}=\mp q_{0}+\omega_{k}^{N}\right)\right\} & = \pm \frac{-\beta \frac{d \omega_{q}}{d q_{0}} e^{\beta\left(\omega_{q} \pm \mu_{N}\right)}}{\left\{e^{\beta\left(\omega_{q} \pm \mu_{N}\right)}+1\right\}^{2}} \\
\lim _{q_{0} \rightarrow 0} \frac{d}{d q_{0}}\left\{ \pm n_{p}^{\mp}\left(\omega_{q}=\mp q_{0}+\omega_{k}^{N}\right)\right\} & = \pm \frac{-(\mp) \beta e^{\beta\left(\omega_{k}^{N} \pm \mu_{N}\right)}}{\left\{e^{\beta\left(\omega_{k}^{N} \pm \mu_{N}\right)}+1\right\}^{2}} \\
& =\beta\left[n_{k}^{\mp}\left(1-n_{k}^{\mp}\right)\right] .
\end{aligned}
$$

Again, in the limiting value of $q_{0}, \vec{q} \rightarrow 0$, Eq. (12) can be simplified to

$$
N^{0}=-I_{N} \frac{16 \vec{k}^{4}}{3} .
$$

Hence, by using the above results, the Eq. (17) becomes

$$
\begin{aligned}
\eta_{N} & =\frac{8 \beta I_{N}}{15} \int \frac{d^{3} k}{(2 \pi)^{3}} \frac{\vec{k}^{4}}{4 \omega_{k}^{N^{2}} \Gamma_{N}}\left[n_{k}^{-}\left(1-n_{k}^{-}\right)+n_{k}^{+}\left(1-n_{k}^{+}\right)\right] \\
& =\frac{\beta I_{N}}{15 \pi^{2}} \int \frac{\vec{k}^{6} d \vec{k}}{\omega_{k}^{N^{2}} \Gamma_{N}}\left[n_{k}^{-}\left(1-n_{k}^{-}\right)+n_{k}^{+}\left(1-n_{k}^{+}\right)\right] .
\end{aligned}
$$

This is the one-loop expression of shear viscosity for the matter with nucleon degrees of freedom in the Kubo approach. Although there are possibilities of an infinite number of ladder-type diagrams, which are supposed to be of same order of magnitude $\left[O\left(1 / \Gamma_{N}\right)\right]$ like the one loop, they will be highly suppressed [42]. As we increase the number of loops, the number of extra thermal distribution functions will also appear in the shear viscosity expression and hence their numerical suppression will successively grow. On this basis, the one-loop results may be considered as leadingorder results. One can derive exactly same expression from relaxation-time approximation in the kinetic theory approach.

\section{CALCULATION OF NUCLEON THERMAL WIDTH}

Now, our next aim is to calculate the thermal width of nucleon $\Gamma_{N}$, which can be estimated from the retarded component of nucleon self-energy $\left(\Sigma^{R}\right)$ at finite temperature and density. Their relation is given by

$$
\Gamma_{N}\left(\vec{k}, T, \mu_{N}\right)=-\operatorname{Im} \Sigma^{R}\left(k_{0}=\omega_{k}^{N}, \vec{k}, T, \mu_{N}\right) .
$$

During the propagation in the hot and dense nuclear matter, nucleons may pass through different $\pi B$ loops, where $B$ stand for different higher-mass baryons including the nucleon itself. In this work, all possible four-star baryon resonances with spin $1 / 2$ and $3 / 2$ are considered. These are $N(980), \Delta(1232), N^{*}(1440), N^{*}(1520), N^{*}(1535), \Delta^{*}(1600)$, $\Delta^{*}(1620), N^{*}(1650), \Delta^{*}(1700), N^{*}(1700), N^{*}(1710)$, and $N^{*}(1720)$, where masses (in $\mathrm{MeV}$ ) of the baryons are given 
inside the brackets. The nucleon self-energy for the $\pi B$ loop is shown in Fig. 1(b) and its 11 component can be expressed as

$$
\begin{aligned}
\Sigma^{11}\left(k, T, \mu_{N}\right)= & -i \int \frac{d^{4} l}{(2 \pi)^{4}} L(k, l) D_{11}\left(l, m_{\pi}, T\right) \\
& \times D_{11}\left(u=k-l, m_{B}, T, \mu_{N}\right),
\end{aligned}
$$

where $D_{11}\left(l, m_{\pi}, T\right)$ and $D_{11}\left(u=k-l, m_{B}, T, \mu_{N}\right)$ are the scalar parts of the pion and baryon propagators at finite temperature and density. $L(k, l)$ contains vertices and the numerator parts of the propagators. The chemical potential of all baryons are assumed to be the same as nucleon chemical potential $\mu_{N}$. Similar to Eq. (8), this 11 component is also related to the retarded component as

$$
\operatorname{Im} \Sigma^{R}(k)=\operatorname{coth}\left\{\frac{\beta\left(k_{0}-\mu_{N}\right)}{2}\right\} \operatorname{Im} \Sigma_{11}(k) .
$$

Performing the $l_{0}$ integration in Eq. (25) and then using the relation (26), we get the imaginary part of retarded self-energy,

$$
\begin{aligned}
\operatorname{Im} \Sigma^{R}(k)= & \pi \int \frac{d^{3} l}{(2 \pi)^{3}} \frac{1}{4 \omega_{l}^{\pi} \omega_{u}^{B}}\left[L ( l _ { 0 } = \omega _ { l } ^ { \pi } , \vec { l } , k ) \left[\left\{1+n_{l}\left(\omega_{l}^{\pi}\right)-n_{u}^{+}\left(k_{0}-\omega_{l}^{\pi}\right)\right\} \delta\left(k_{0}-\omega_{l}^{\pi}-\omega_{u}^{B}\right)\right.\right. \\
& \left.+\left\{-n_{l}\left(\omega_{l}^{\pi}\right)-n_{u}^{-}\left(-k_{0}+\omega_{l}^{\pi}\right)\right\} \delta\left(k_{0}-\omega_{l}^{\pi}+\omega_{u}^{B}\right)\right]+L\left(l_{0}=-\omega_{l}^{\pi}, \vec{l}, k\right)\left[\left\{n_{l}\left(\omega_{l}^{\pi}\right)+n_{u}^{+}\left(k_{0}+\omega_{l}^{\pi}\right)\right\} \delta\left(k_{0}+\omega_{l}^{\pi}-\omega_{u}^{B}\right)\right. \\
& \left.\left.+\left\{-1-n_{l}\left(\omega_{l}^{\pi}\right)+n_{u}^{-}\left(-k_{0}-\omega_{l}^{\pi}\right)\right\} \delta\left(k_{0}+\omega_{l}^{\pi}+\omega_{u}^{B}\right)\right]\right],
\end{aligned}
$$

where $\omega_{u}^{B}=\left[(\vec{k}-\vec{l})^{2}+m_{B}^{2}\right]^{1 / 2}, n_{u}^{ \pm}$and $n_{l}$ are respectively Fermi-Dirac and Bose-Einstein distribution functions. The regions of different branch cuts in the $k_{0}$ axis are $\left[-\infty\right.$ to $\left.-\left[\vec{k}^{2}+\left(m_{\pi}+m_{B}\right)^{2}\right]^{1 / 2}\right]$ for a unitary cut in the negative $k_{0}$ axis, $[-$ $\left[\vec{k}^{2}+\left(m_{B}-m_{\pi}\right)^{2}\right]^{1 / 2}$ to $\left.\left[\vec{k}^{2}+\left(m_{B}-m_{\pi}\right)^{2}\right]^{1 / 2}\right]$ for a Landau cut and $\left[\vec{k}^{2}+\left(m_{\pi}+m_{B}\right)^{2}\right]^{1 / 2}$ to $\left.\infty\right]$ for a unitary cut in the positive $k_{0}$ axis. These represent the different kinematic regions where the imaginary part of the nucleon self-energy becomes nonzero because of the different $\delta$ functions in Eq. (27). The $\Gamma_{N}$ for all $\pi B$ loops (except the $\pi N$ ) are coming from the Landau-cut contribution associated with the third term of Eq. (27), which can be simplified as

$$
\Gamma_{N}=\frac{1}{16 \pi \vec{k}} \int_{\widetilde{\omega}^{+}}^{\widetilde{\omega}^{-}} d \widetilde{\omega}\left\{n_{l}(\widetilde{\omega})+n_{u}^{+}\left(\omega_{k}^{N}+\widetilde{\omega}\right)\right\} L\left(l_{0}=-\widetilde{\omega}, \vec{l}=\sqrt{\widetilde{\omega}^{2}-m_{\pi}^{2}}, k_{0}=\omega_{k}^{N}, \vec{k}\right)
$$

where $n_{l}(\widetilde{\omega})=1 /\left\{e^{\beta \widetilde{\omega}}-1\right\}, n_{u}^{+}\left(\omega_{k}^{N}+\widetilde{\omega}\right)=1 /\left\{e^{\beta\left(\widetilde{\omega}+\omega_{k}^{N}-\mu_{N}\right)}+1\right\}, \widetilde{\omega}^{ \pm}=\frac{R^{2}}{2 m_{N}^{2}}\left(-\omega_{k}^{N} \pm \vec{k} W\right)$ with $W=\left(1-4 m_{\pi}^{2} m_{N}^{2} / R^{4}\right)^{1 / 2}$ and $R^{2}=m_{N}^{2}+m_{\pi}^{2}-m_{B}^{2}$.

The effective Lagrangian densities for $B N \pi$ interactions are [46]

$$
\mathcal{L}=\frac{f}{m_{\pi}} \bar{\psi}_{B} \gamma^{\mu}\left\{\begin{array}{c}
i \gamma^{5} \\
\mathbb{1}
\end{array}\right\} \psi_{N} \partial_{\mu} \pi+\text { H.c. } \quad \text { for } \quad J_{B}^{P}=\frac{1^{ \pm}}{2}, \quad \mathcal{L}=\frac{f}{m_{\pi}} \bar{\psi}_{B}^{\mu}\left\{\begin{array}{c}
\mathbb{1} \\
i \gamma^{5}
\end{array}\right\} \psi_{N} \partial_{\mu} \pi+\text { H.c. } \quad \text { for } \quad J_{B}^{P}=\frac{3}{2}^{ \pm},
$$

where coupling constants $f / m_{\pi}$ for different baryons have been fixed from their experimental vacuum widths in $N \pi$ channel. With the help of the above Lagrangian densities, one can easily find

$$
\begin{aligned}
L(k, l)= & -\left(\frac{f}{m_{\pi}}\right)^{2} l\left(k-l-P m_{B}\right) l \quad \text { for } \quad J_{B}^{P}=\frac{1}{2}^{ \pm} \\
L(k, l)= & -\left(\frac{f}{m_{\pi}}\right)^{2}\left(k-l+P m_{B}\right) l_{\mu} l_{\nu}\left\{-g^{\mu \nu}+\frac{1}{3} \gamma^{\mu} \gamma^{\nu}+\frac{2}{3 m_{B}^{2}}(k-l)^{\mu}(k-l)^{v}\right. \\
& \left.+\frac{1}{3 m_{B}}\left(\gamma^{\mu}(k-l)^{\nu}-(k-l)^{\mu} \gamma^{\nu}\right)\right\} \quad \text { for } J_{B}^{P}=\frac{3^{ \pm}}{2} .
\end{aligned}
$$

For simplification, the coefficients of $\gamma^{0}$ and $\mathbb{1}$ are taken from Ref. [47] and their addition gives

$$
\begin{aligned}
& L(k, l)=-\left(\frac{f}{m_{\pi}}\right)^{2}\left\{\left(\frac{R^{2}}{2}-m_{\pi}^{2}\right) l_{0}-P m_{\pi}^{2} m_{B}\right\} \quad \text { for } \quad J_{B}^{P}=\frac{1^{ \pm}}{2}, \\
& L(k, l)=-\left(\frac{f}{m_{\pi}}\right)^{2} \frac{2}{3 m_{B}^{2}}\left\{\left(\frac{R^{2}}{2}-m_{\pi}^{2}\right)^{2}-m_{\pi}^{2} m_{B}^{2}\right\}\left(k_{0}-l_{0}+P m_{B}\right) \quad \text { for } \quad J_{B}^{P}=\frac{3^{ \pm}}{2} .
\end{aligned}
$$

The isospin part of the Lagrangian densities are not written in Eq. (29). The isospin structure for $J_{B}^{P}=\frac{1}{2}^{ \pm}$and $J_{B}^{P}=\frac{3}{2}^{ \pm}$ should be $\bar{\psi} \vec{\tau} \cdot \vec{\pi} \psi$ and $\vec{\psi} \vec{T} \cdot \vec{\pi} \psi$, respectively, where $\vec{T}$ is the spin $3 / 2$ transition operator and $\vec{\tau}$ is the Pauli operator. This issue is managed by multiplying appropriate isospin factors with the expressions of the corresponding loop diagrams. The 
isospin factor for the $\pi N$ or $\pi N^{*}$ loop is $I_{N \rightarrow \pi N, N^{*}}=3$, whereas for the $\pi \Delta$ or $\pi \Delta^{*}$ loop, $I_{N \rightarrow \pi \Delta, \Delta^{*}}=2$.

All baryon resonances have finite vacuum width in the $N \pi$ decay channel. The calculations of these decay widths are very essential in the present work for two reasons. First is to fix the coupling constants $f / m_{\pi}$ for different $B N \pi$ interaction Lagrangian densities and second is to include the effect of these baryon widths $\left(\Gamma_{B}\right)$ on the nucleon thermal width $\Gamma_{N}$. By using the Lagrangian densities, the vacuum decay width of baryons $B$ for $N \pi$ channel can be obtained as

$$
\begin{aligned}
\Gamma_{B}\left(m_{B}\right)= & \frac{I_{N^{*} \rightarrow \pi N}}{2 J_{B}+1}\left(\frac{f}{m_{\pi}}\right)^{2} \frac{\left|\vec{p}_{\mathrm{c} . \mathrm{m} .}\right|}{2 \pi m_{B}}\left[2 m_{B}\left|\vec{p}_{\mathrm{c} . \mathrm{m} .}\right|^{2}\right. \\
& \left.+m_{\pi}^{2}\left(\omega_{\mathrm{c} . \mathrm{m} .}^{N}-P m_{N}\right)\right] \text { for } J_{B}^{P}=\frac{1^{ \pm}}{2}, \\
\Gamma_{B}\left(m_{B}\right)= & \frac{I_{\Delta, \Delta^{*} \rightarrow \pi N}}{2 J_{B}+1}\left(\frac{f}{m_{\pi}}\right)^{2} \frac{\mid \vec{p}_{\mathrm{c} . \mathrm{m} .\left.\right|^{3}}}{3 \pi m_{B}} \\
& \times\left[\omega_{\mathrm{c} . \mathrm{m} .}^{N}+P m_{N}\right] \text { for } J_{B}^{P}=\frac{3^{ \pm}}{2},
\end{aligned}
$$

where $\quad\left|\vec{p}_{\text {c.m. }}\right|=\left[\left\{m_{B}^{2}-\left(m_{N}+m_{\pi}\right)^{2}\right\}\left\{m_{B}^{2}-\left(m_{N}\right.\right.\right.$ $\left.\left.\left.-m_{\pi}\right)^{2}\right\}\right]^{1 / 2} /\left(2 m_{B}\right)$ and $\omega_{\mathrm{c} . \mathrm{m} .}^{N}=\left(\left|\vec{p}_{\mathrm{c} . \mathrm{m} .}\right|^{2}+m_{N}^{2}\right)^{1 / 2}$. The isospin factors are $I_{N^{*} \rightarrow \pi N}=3$ and $I_{\Delta, \Delta^{*} \rightarrow \pi N}=1$ for the $N \pi$ decay channels of $N^{*}$ and $\Delta^{*}$ (or $\Delta$ ), respectively.

Now, $\Gamma_{N}$ in Eq. (28) can be convoluted (see, e.g., Refs. $[48,49])$ as

$$
\begin{aligned}
\Gamma_{N}\left(m_{B}\right) & =\frac{1}{N_{B}} \int_{m_{B}-2 \Gamma_{B}\left(m_{B}\right)}^{m_{B}+2 \Gamma_{B}\left(m_{B}\right)} d M_{B} A_{B}\left(M_{B}\right) \Gamma_{N}\left(M_{B}\right), \\
N_{B} & =\int_{m_{B}-2 \Gamma_{B}\left(m_{B}\right)}^{m_{B}+2 \Gamma_{B}\left(m_{B}\right)} A_{B}\left(M_{B}\right),
\end{aligned}
$$

where

$$
A_{B}\left(M_{B}\right)=\frac{1}{\pi} \operatorname{Im}\left[\frac{1}{M_{B}-m_{B}+i \Gamma_{B}\left(M_{B}\right) / 2}\right]
$$

TABLE I. From the left to right columns, the table contain the baryons, their spin-parity quantum numbers $J_{B}^{P}$, isospin $I_{B}$, total decay width $\Gamma_{\text {tot }}$, decay width in $N \pi$ channels $\Gamma_{B \rightarrow N \pi}$ or $\Gamma_{B}\left(m_{B}\right)$ in Eq. (32) (brackets displaying its branching ratio), and, finally, the coupling constants $f / m_{\pi}$.

\begin{tabular}{lccccc}
\hline \hline Baryons & $J_{B}^{P}$ & $I_{B}$ & $\Gamma_{\text {tot }}$ & $\Gamma_{B \rightarrow N \pi}($ B.R. $)$ & $f / m_{\pi}$ \\
\hline$\Delta^{(1232)}$ & $\frac{3}{2}^{+}$ & $3 / 2$ & 0.117 & $0.117(100 \%)$ & 15.7 \\
$N^{*}(1440)$ & $\frac{1}{2}^{+}$ & $1 / 2$ & 0.300 & $0.195(65 \%)$ & 2.5 \\
$N^{*}(1520)$ & $\frac{3}{2}^{-}$ & $1 / 2$ & 0.115 & $0.069(60 \%)$ & 11.6 \\
$N^{*}(1535)$ & $\frac{1}{2}^{-}$ & $1 / 2$ & 0.150 & $0.068(45 \%)$ & 1.14 \\
$\Delta^{*}(1600)$ & $\frac{3}{2}^{+}$ & $3 / 2$ & 0.320 & $0.054(17 \%)$ & 3.4 \\
$\Delta^{*}(1620)$ & $\frac{1}{2}^{-}$ & $3 / 2$ & 0.140 & $0.035(25 \%)$ & 1.22 \\
$N^{*}(1650)$ & $\frac{1}{2}^{-}$ & $1 / 2$ & 0.150 & $0.105(70 \%)$ & 1.14 \\
$\Delta^{*}(1700)$ & $\frac{3}{2}^{-}$ & $3 / 2$ & 0.300 & $0.045(15 \%)$ & 9.5 \\
$N^{*}(1700)$ & $\frac{3}{2}^{-}$ & $1 / 2$ & 0.100 & $0.012(12 \%)$ & 2.8 \\
$N^{*}(1710)$ & $\frac{1}{2}^{+}$ & $1 / 2$ & 0.100 & $0.012(12 \%)$ & 0.35 \\
$N^{*}(1720)$ & $\frac{3}{2}^{+}$ & $1 / 2$ & 0.250 & $0.028(11 \%)$ & 1.18 \\
\hline \hline
\end{tabular}

is the vacuum spectral function of baryons for their vacuum decay width in the $N \pi$ channel. Replacing the baryon mass $m_{B}$ by its invariant mass $M_{B}$ in Eq. (32), one can get the off-mass shell expression of $\Gamma_{B}(M)$. The values of the coupling constants $f / m_{\pi}$, which are fixed from the experimental values of the baryon decay width in $N \pi$ channels [50], are shown in Table I.

\section{RESULTS AND DISCUSSION}

Let us first take a glance at the invariant mass distribution of the imaginary part of the nucleon self-energy for different $\pi B$ loops. Figure 2 shows the results for baryons $B=$ $N(940), \Delta(1232)$ (upper panel) and $B=\Delta^{*}(1620), N^{*}(1650)$, $N^{*}(1720)$ (lower panel), whereas Fig. 3 displays the results for baryons $B=N^{*}(1440), N^{*}(1520), \Delta^{*}(1600)$ (upper panel) and $B=N^{*}(1535), \Delta^{*}(1700)$ (lower panel). The numerical strengths for $B=N^{*}(1700)$ and $N^{*}(1710)$ are too low to display with the other baryons. These results are obtained by replacing $\omega_{k}^{N}=\left(\vec{k}^{2}+m_{N}^{2}\right)^{1 / 2}$ by $\omega_{k}=\left(\vec{k}^{2}+M^{2}\right)^{1 / 2}$ in Eqs. (28) (dashed line) and (33) (solid line) for the fixed values of $\vec{k}=0, \mu_{N}=0$, and $T=0.150 \mathrm{GeV}$. From the sharp ending of the dashed line, the Landau regions for different loops are clearly visible. As an example for the $\pi N$ loop for the Landau region is $M=0$ to $m_{N}-m_{\pi}$, i.e., 0 to $0.8 \mathrm{GeV}$. Due to the folding of the baryon spectral functions, these sharp endings are smeared towards higher values of $M$. Since $\Sigma^{R}(M)$ also depends on $T, \mu_{N}$, and $\vec{k}$, the total contribution of $\Sigma^{R}(M)$ from all loops has been shown in Fig. 4 for different sets of $T$, $\mu_{N}$, and $\vec{k}$.

The nucleon thermal width $\Gamma_{N}$ is basically the contribution of $\operatorname{Im} \Sigma^{R}$ at $M=m_{N}$, which is marked by dotted line. Being an on-shell quantity, $\Gamma_{N}$ is associated with the thermodynamical probability of different on-shell scattering processes instead

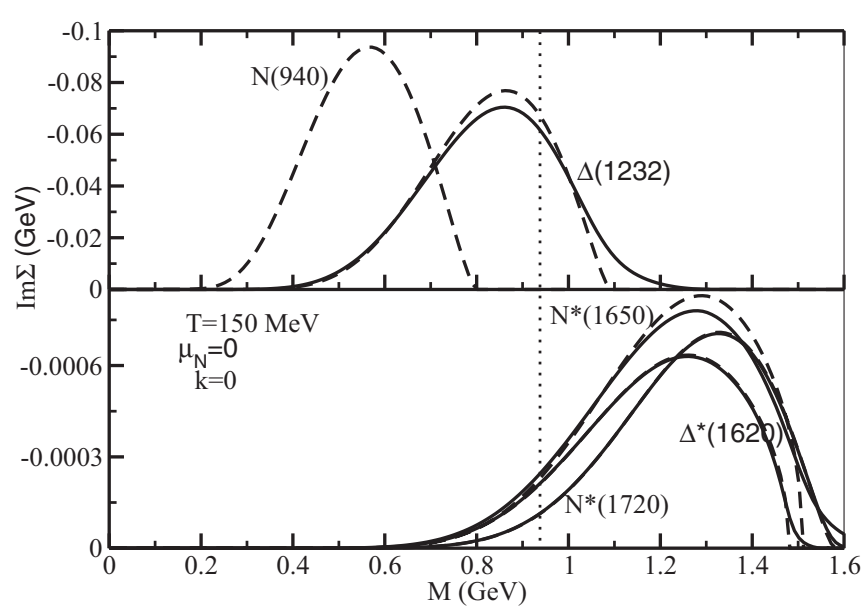

FIG. 2. Imaginary part of nucleon self-energy for different $\pi B$ loops are individually shown before (dashed line) and after (solid line) folding by corresponding baryon spectral functions. $B=N(940), \Delta(1232)$ are in upper panel whereas $B=\Delta^{*}(1620)$, $N^{*}(1650), N^{*}(1720)$ are in lower panel for fixed values of threemomentum of $N(\vec{k}=0)$, temperature $(T=0)$, and baryon chemical potential $\left(\mu_{N}=0\right)$. 


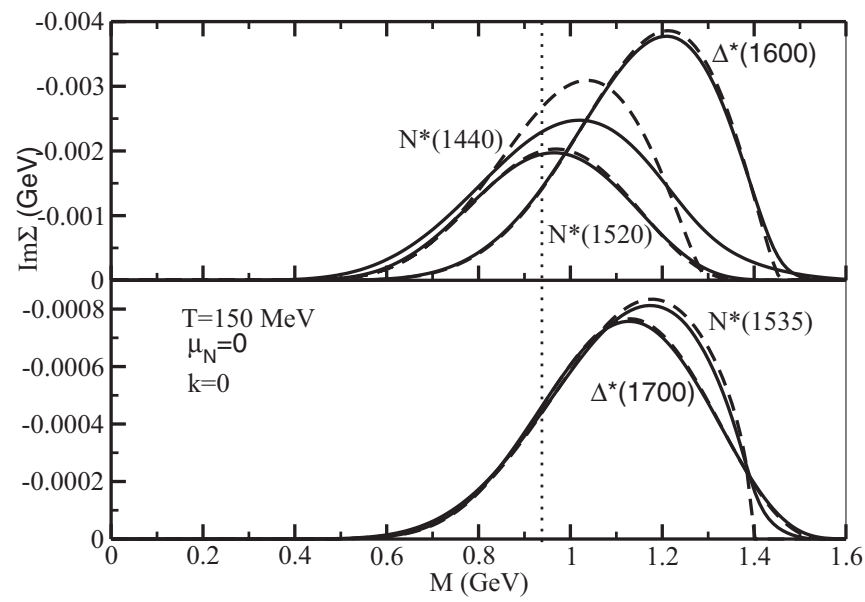

FIG. 3. Same as Fig. 2 for the rest of the baryons $B=N^{*}(1440)$, $N^{*}(1520), \Delta^{*}(1600)$ (upper panel) and $B=N^{*}(1535), \Delta^{*}(1700)$ (lower panel).

of off-shell scattering processes as described by Weldon for the imaginary part of the self-energy in Ref. [51]. Following Weldon's prescription, forward and inverse scattering of nucleons can be respectively described as follows: During propagation of $N$, it can disappear by absorbing a thermalized $\pi$ from the medium to create a thermalized $B$. Again $N$ can appear by absorbing a thermalized $B$ from the medium as well as by emitting a thermalized $\pi$. The $n_{l}\left(1-n_{u}^{+}\right)$and $n_{u}^{+}\left(1+n_{l}\right)$ are the corresponding statistical probabilities of the forward and inverse scattering, respectively [51], because just by adding them, we get the thermal-distribution part of Eq. (28), i.e., $\left(n_{l}+n_{u}^{+}\right)$.

From Eq. (28) or (33), we see that $\Gamma_{N}$ depends on the temperature $T$, the baryon chemical potential $\mu_{N}$, and the three-momentum $\vec{k}$ of the nucleon. The upper panels of Figs. 5 and 6 are, respectively, displaying the variation of $\Gamma_{N}$ with $T$ for different sets of $\left(\vec{k}, \mu_{N}\right)$ and of $\Gamma_{N}$ with $\mu_{N}$ for different set of $(\vec{k}, T)$. The mean-free path can be defined as $\lambda_{N}\left(\vec{k}, T, \mu_{N}\right)=$

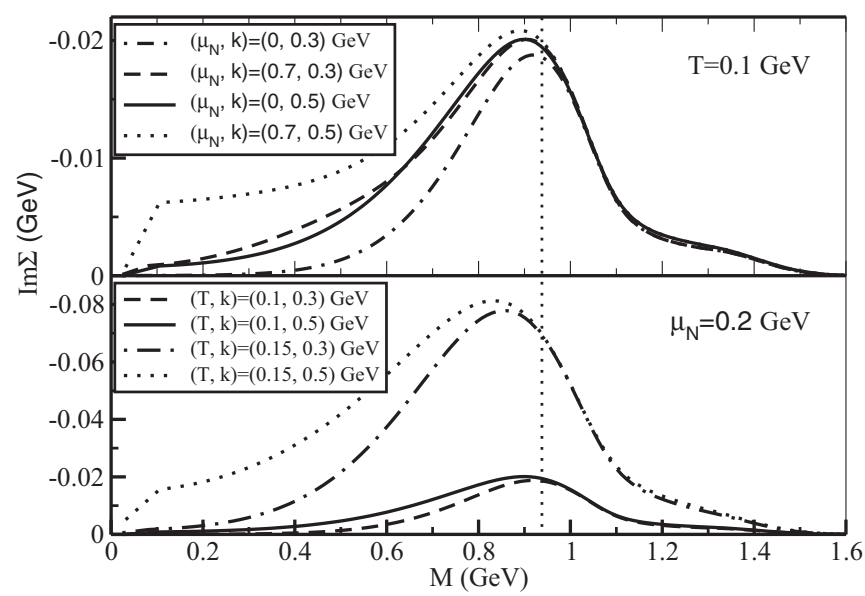

FIG. 4. Imaginary part of total self-energy for different sets of nucleon momentum $(\vec{k})$, temperature $(T)$, and baryon chemical potential $\left(\mu_{N}\right)$.

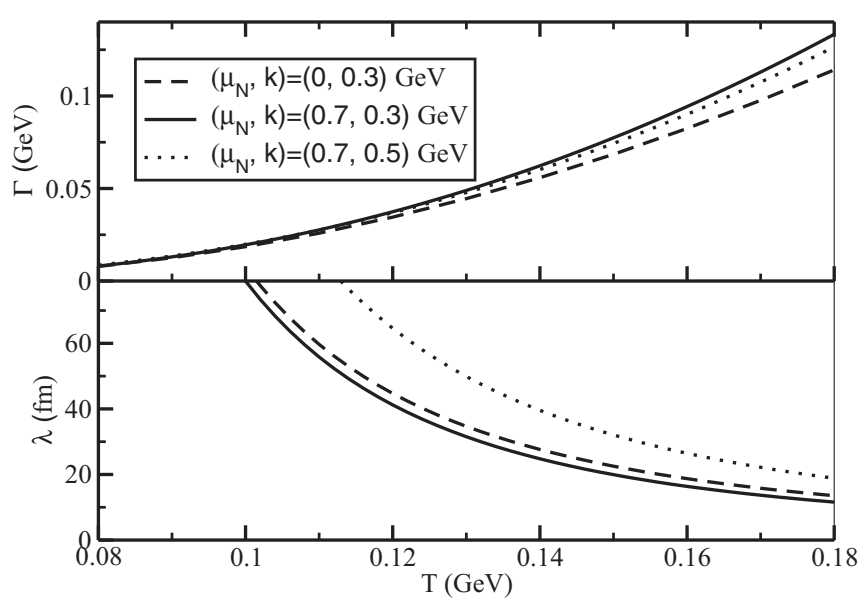

FIG. 5. The variation of nucleon thermal width $\Gamma_{N}$ (upper panel) and its corresponding mean-free path $\lambda_{N}$ (lower panel) with $T$.

$\vec{k} /\left[\omega_{k}^{N} \Gamma_{N}\left(\vec{k}, T, \mu_{N}\right)\right]$ and its corresponding variation with $T$ and $\mu_{N}$ are respectively shown in the lower panels of Figs. 5 and 6. The range of $T$ and $\mu_{N}$, in which $\lambda_{N}$ is smaller than the dimension of the medium $(\sim 10-40 \mathrm{fm}$, a typical dimension of strongly interacting matter, produced in the laboratories of HIC), plays the main role of dissipation via scattering in the medium because the larger $\lambda$ is associated with the scenario after freeze-out of the medium. From the dashed line of Fig. 5 we see that $T>0.120 \mathrm{GeV}$ (but up to $T_{c} \approx 0.175 \mathrm{GeV}$ ) is the relevant region for baryon-free nuclear matter $\left(\mu_{N}=0\right)$. Whereas for finite baryon chemical potential (e.g., solid line of Fig. 5 at $\mu_{N}=0.7 \mathrm{GeV}$ ), this relevant $T$ region will be shifted slightly toward lower temperature (in addition, $T_{c}$ is also expected to decrease with increase of $\mu_{N}$ ). Since high momentum $(\vec{k})$ of constituent particles always helps them to freeze-out from the medium, the relevant $T$ region for nucleon with high $\vec{k}$ is reduced by shifting towards the high $T$ region.

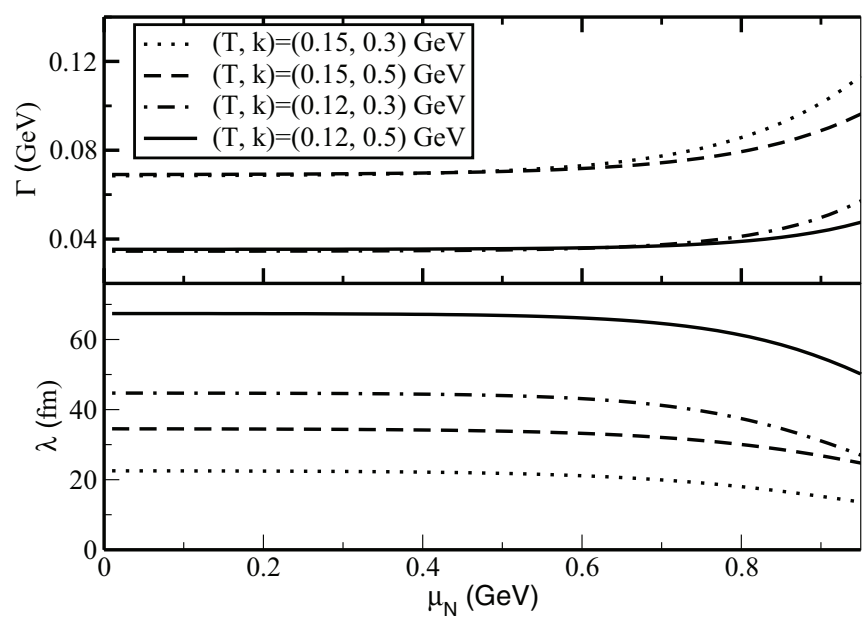

FIG. 6. The variation of nucleon thermal width $\Gamma_{N}$ (upper panel) and its corresponding mean-free path $\lambda_{N}$ (lower panel) as a function of baryon chemical potential $\mu_{N}$. 


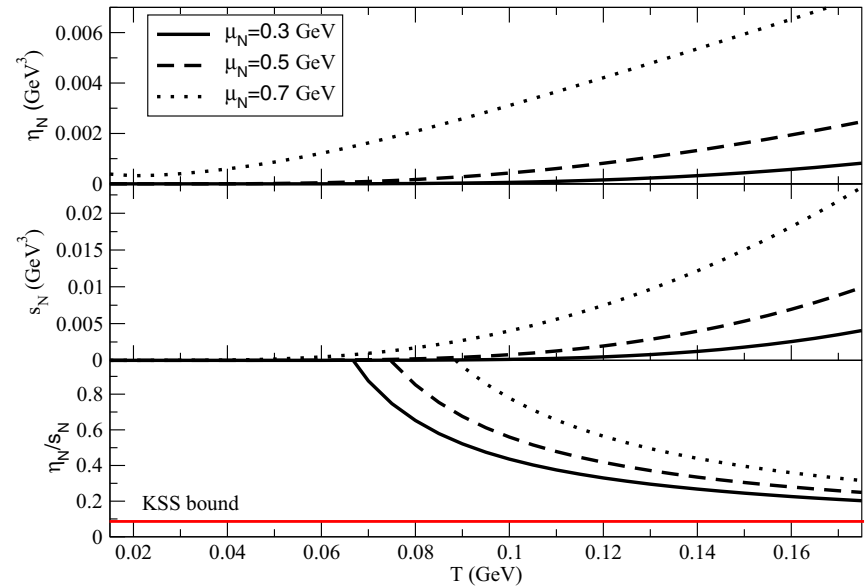

FIG. 7. (Color online) The $T$ dependence of $\eta_{N}$ (upper panel), $s_{N}$ (middle panel), and $\eta_{N} / s_{N}$ (lower panel) of the nucleonic component. The straight red line denotes the KSS bound.

This can be understood by comparing the solid and dotted lines in the lower panel of Fig. 5.

Using the numerical function $\Gamma_{N}\left(\vec{k}, T, \mu_{N}\right)$ in Eq. (23), we get $\eta_{N}$ as a function of $T$ and $\mu_{N}$, which is shown in the upper panels of Figs. 7 and 8. Here we see $\eta_{N}$ is monotonically increasing with both $T$ and $\mu_{N}$. By using the simple equilibrium expression of entropy density $\left(s_{N}\right)$ for nucleons,

$$
s_{N}=4 \beta \int \frac{d^{3} \vec{k}}{(2 \pi)^{3}}\left(\omega_{k}^{N}+\frac{\vec{k}^{2}}{3 \omega_{k}^{N}}-\mu_{N}\right) n_{k}^{+}\left(\omega_{k}^{N}\right),
$$

the ratio $\eta_{N} / s_{N}$ has been generated as a function of $T$ and $\mu_{N}$. From the lower panels of Figs. 7 and 8, we see that $\eta_{N} / s_{N}$ can be reduced by increasing $T$ as well as by decreasing $\mu_{N}$.

In the left and right panels of Fig. 9, the contributions of different loops (dominating loops only) are individually shown in $\eta_{N}$ vs $T$ and $\eta_{N}$ vs $\mu_{N}$ graphs, respectively. The $\pi \Delta$ loop

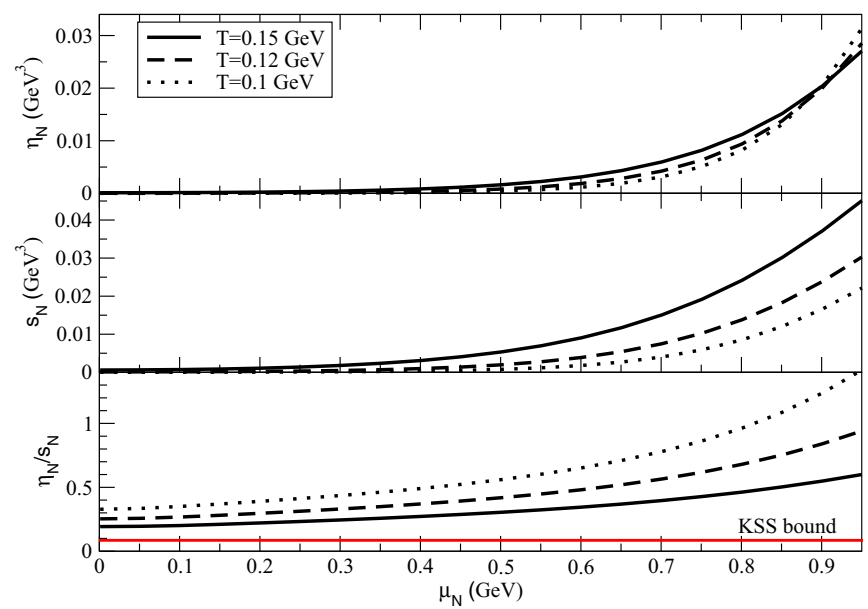

FIG. 8. (Color online) The variation of $\eta_{N}$ (upper panel), $s_{N}$ (middle panel), and $\eta_{N} / s_{N}$ (lower panel) of the nucleonic component with $\mu_{N}$.

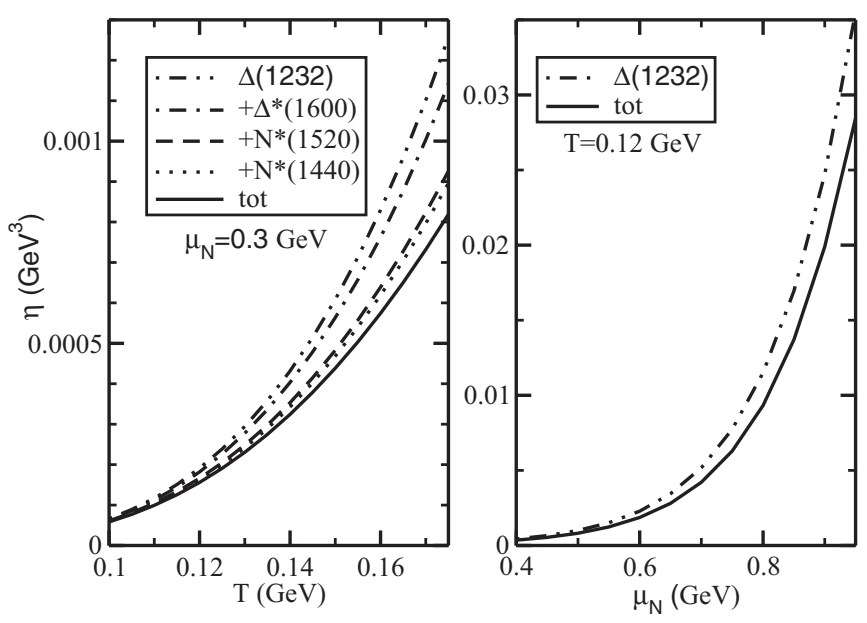

FIG. 9. The contributions of different $\pi B$ loops in $\eta_{N}(T)$ (left panel) and $\eta_{N}\left(\mu_{N}\right)$ (right panel).

plays a leading role to generate the typical values $(0.0001-0.01$ $\mathrm{GeV}^{3}$ ) of $\eta_{N}$ for strongly interacting matter because the major part of the nucleon thermal width is coming from this loop only.

Up to now, we calculated the contribution of shear viscosity from the nucleon thermal width, although a major contribution comes from the thermal width of pions. Hence, one should add the pionic contribution to the nucleon contribution to get total shear viscosity of nuclear matter at finite temperature and density. In our recent work [52], the shear viscosity, coming from the pionic thermal width, has already been addressed. The one-loop Kubo expression of shear viscosity and the ideal expression of entropy density for pionic components are respectively given below:

$$
\begin{gathered}
\eta_{\pi}=\frac{\beta}{10 \pi^{2}} \int \frac{d^{3} k \vec{k}^{6}}{\Gamma_{\pi} \omega_{k}^{\pi 2}} n_{k}\left(\omega_{k}^{\pi}\right)\left[1+n_{k}\left(\omega_{k}^{\pi}\right)\right], \\
s_{\pi}=3 \beta \int \frac{d^{3} \vec{k}}{(2 \pi)^{3}}\left(\omega_{k}^{\pi}+\frac{\vec{k}^{2}}{3 \omega_{k}^{\pi}}\right) n_{k}\left(\omega_{k}^{\pi}\right),
\end{gathered}
$$

where $n_{k}\left(\omega_{k}^{\pi}\right)=1 /\left\{e^{\beta \omega_{k}^{\pi}}-1\right\}$ is the Bose-Einstein distribution function for pions with $\omega_{k}^{\pi}=\left(\vec{k}^{2}+m_{\pi}^{2}\right)^{1 / 2}$, and $\Gamma_{\pi}$ is the thermal width of $\pi$ mesons in the medium due to $\pi \sigma$ and $\pi \rho$ fluctuations.

Now, adding that pion contribution with the nucleon, one can simply get the total shear viscosity of nuclear matter as

$$
\eta_{\mathrm{tot}}=\eta_{\pi}+\eta_{N}
$$

where $\eta_{\pi}$ and $\eta_{N}$ do not face any mixing effects of pion density, $\rho_{\pi}=3 \int \frac{d^{3} k}{(2 \pi)^{3}} n_{k}\left(\omega_{k}^{\pi}\right)$ and nucleon density, $\rho_{N}=4 \int \frac{d^{3} k}{(2 \pi)^{3}} n_{k}^{+}\left(\omega_{k}^{N}\right)$. However, the viscosity of a singlecomponent gas should be different from the viscosity of that component in a mixed gas [24,53]. To incorporate this mixing effect for a rough estimation, we follow the approximated relation $[24,53]$

$$
\eta_{\mathrm{tot}}^{\operatorname{mix}}=\eta_{\pi}^{\mathrm{mix}}+\eta_{N}^{\mathrm{mix}}
$$




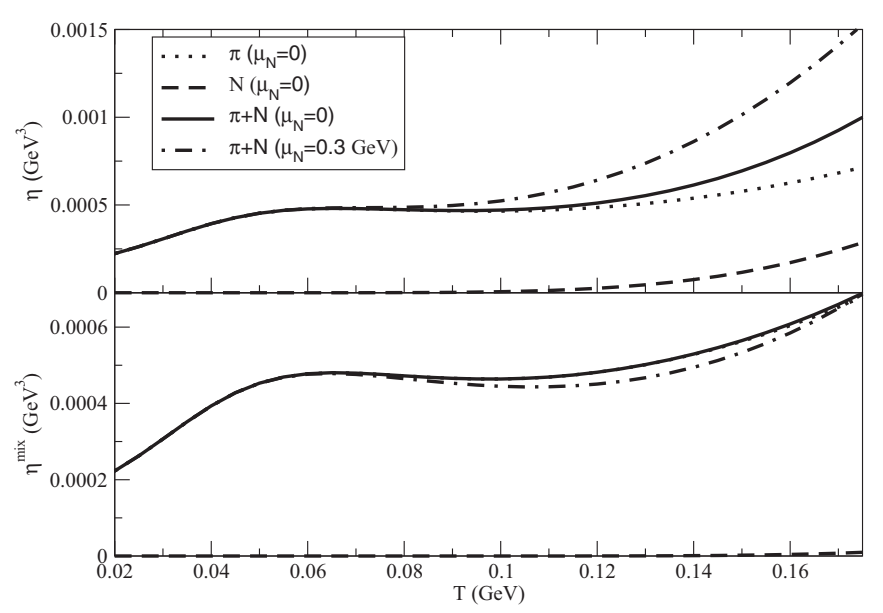

FIG. 10. The $T$ dependence of shear viscosity for pionic (dotted line) and nucleonic (dashed line) components and their total at $\mu_{N}=$ 0 (solid line) and $\mu_{N}=0.3 \mathrm{GeV}$ (dash-dotted line). The upper and lower panels contain the results without and with the mixing effect, obtained from Eqs. (38) and (39), respectively.

where

$$
\begin{aligned}
\eta_{\pi}^{\operatorname{mix}} & =\frac{\eta_{\pi}}{1+\left(\frac{\rho_{N}}{\rho_{\pi}}\right)\left(\frac{\sigma_{\pi N}}{\sigma_{\pi \pi}}\right) \sqrt{\frac{1+m_{\pi} / m_{N}}{2}}} \\
\eta_{N}^{\operatorname{mix}} & =\frac{\eta_{N}}{1+\left(\frac{\rho_{\pi}}{\rho_{N}}\right)\left(\frac{\sigma_{\pi N}}{\sigma_{N N}}\right) \sqrt{\frac{1+m_{N} / m_{\pi}}{2}}} .
\end{aligned}
$$

For simplicity, the cross sections of all kinds of scattering are taken as constant with the same order of magnitude (i.e., $\left.\sigma_{\pi \pi} \approx \sigma_{\pi N} \approx \sigma_{N N}\right)$. In the upper panels of Figs. 10 and 11, the $T$ and $\mu_{N}$ dependence of $\eta_{\pi}$ (dotted line), $\eta_{N}$ (dashed line), and their total $\eta_{\text {tot }}$ (solid line and dash-dotted line for two different values of $\mu_{N}$ and $T$ ) are separately shown. Whereas the lower panels of the figures show their corresponding mixing effect following from Eqs. (40), (41), and (39). From Fig. 11, one should notice that the independent nature of $\eta_{\pi}\left(\mu_{N}\right)$ has been

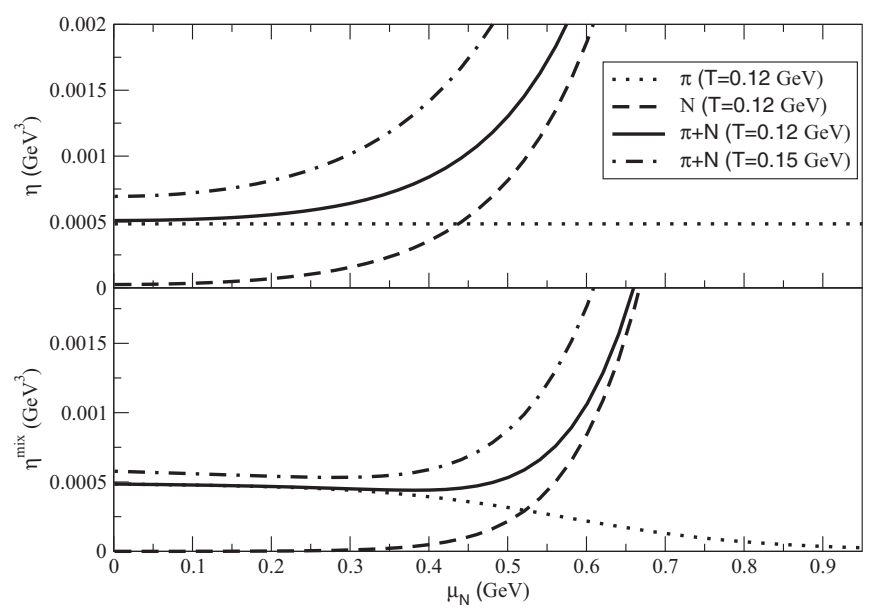

FIG. 11. Corresponding results of Fig. 10 against the $\mu_{N}$ axis with two different temperatures.

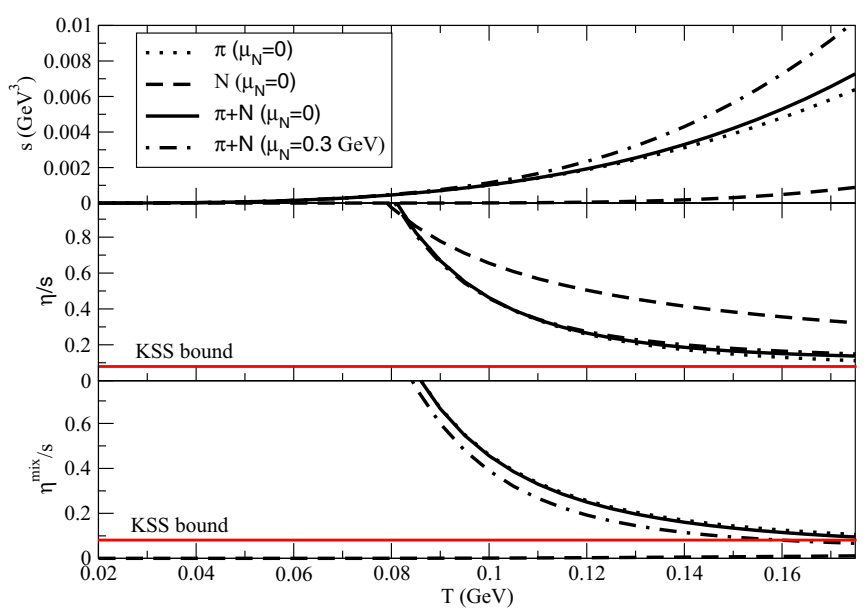

FIG. 12. (Color online) The entropy density (upper panel), viscosity to entropy density ratio without (middle panel) and with (lower panel) the mixing effect as functions of $T$.

changed to a decreasing function due to the mixing effect. A similar qualitative trend was seen in Ref. [24].

The entropy density of the nucleon component from Eq. (35), the pion component from Eq. (37), and their total $s_{\text {tot }}=s_{N}+s_{\pi}$ are individually shown in the upper panels of Figs. 12 and 13 as functions of $T$ and $\mu_{N}$, respectively. The corresponding $\eta / s$ without (middle panel) and with (lower panel) the mixing effect are shown in Figs. 12 and 13 as a function of $T$ and $\mu_{N}$, respectively. The decreasing nature of the total $\eta / s(T)$ qualitatively remains the same after incorporating the mixing effect, whereas an increasing function of the total $\eta / s\left(\mu_{N}\right)$ transforms to a decreasing function due to this mixing effect. Comparing our results with the results of Itakura et al. [24], where $\eta / s\left(\mu_{N}\right)$ also decreases with $\mu_{N}$, the mixing effect appears to be very important. However, the total $\eta / s\left(\mu_{N}\right)$ in the mixing scenario becomes an increasing function beyond $\mu_{N} \approx 0.5 \mathrm{GeV}$ because the increasing rate of $\eta_{N}^{\operatorname{mix}}\left(\mu_{N}\right)$ dominates over the decreasing

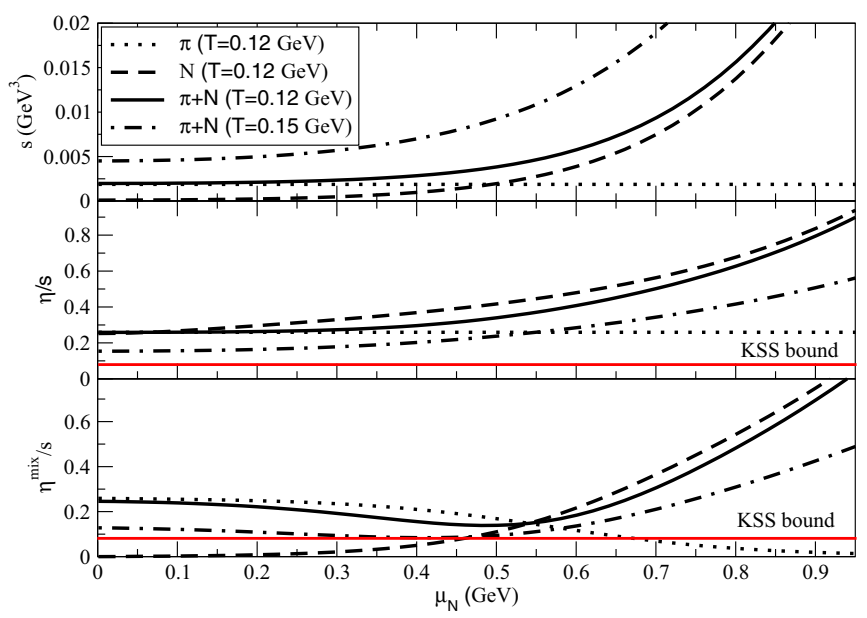

FIG. 13. (Color online) The corresponding results of Fig. 12 are shown with respect to $\mu_{N}$. 
rate of $\eta_{\pi}^{\operatorname{mix}}\left(\mu_{N}\right)$ in that region. Using the effective hadronic Lagrangian, the conclusion of our results should be concentrated within the regions of $0.100 \mathrm{GeV}<T<0.160 \mathrm{GeV}$ and $0<\mu_{N}<0.500 \mathrm{GeV}$.

\section{SUMMARY AND CONCLUSION}

Owing to the Kubo relation, the shear viscosity can be expressed in terms of a two-point function of the viscous stress tensors at finite temperature. By using the real-time thermal field theoretical method, this two-point function has been represented as an $N N$ loop diagram when the nucleons are considered as constituent particles of the medium. A finite nucleon thermal width $\Gamma_{N}$ was traditionally included in the nucleon propagators of the $N N$ loop for getting a nondivergent shear viscosity $\eta_{N}$. This nucleon thermal width is obtained from the one-loop self-energy of nucleons at finite temperature and density. Different possible pion-baryon loops are considered to calculate the total $\Gamma_{N}$, which depends on the three-momentum of nucleons $(\vec{k})$ as well as the medium parameters $T$ and $\mu_{N}$. By using the numerical function $\Gamma_{N}\left(\vec{k}, T, \mu_{N}\right), \eta_{N}$ and $\eta_{N} / s_{N}$ are numerically generated as functions of $T$ and $\mu_{N}$. Adding the pionic contribution taken from Ref. [52] with the numerical values of the nucleonic component, we obtained the total shear viscosity, where a gross mixing effect of a two-component system was implemented. Along the temperature axis, the shear viscosity of both pion and nucleon components appear as an increasing function, whereas along the $\mu_{N}$ axis the shear viscosity of the pion component changes from its constant behavior to a decreasing function due to the presence of the mixing effect. The total shear viscosity to entropy density ratio $\left(\eta_{\mathrm{tot}}^{\operatorname{mix}} / s_{\mathrm{tot}}\right)$ for the pion-nucleon mixed gas reduces with increasing $T$ as well as $\mu_{N}$ and quantitatively becomes very close to the KSS bound. This behavior indicates that $\eta_{\text {tot }}^{\text {mix }} / s_{\text {tot }}$ tends to reach its minimum value near the transition temperature at vanishing as well as finite values of $\mu_{N}$. According to these results, the finite baryon chemical potential helps the nuclear matter to come closer to its (nearly) perfect fluid nature.

\section{ACKNOWLEDGMENTS}

The work was financially supported by the Fundacao de Amparo a Pesquisa do Estado de Sao Paulo, FAPESP (Brazilian agencies) under Contract No. 2012/16766-0. I am very grateful to Professor Gastao Krein for his academic and nonacademic support during my postdoctoral period in Brazil. I would also like to thank Abhishek Mishra, Sandeep Gautam, and Supriya Mondal for their useful help while writing this article.
[1] P. Romatschke and U. Romatschke, Phys. Rev. Lett. 99, 172301 (2007); M. Luzum and P. Romatschke, Phys. Rev. C 78, 034915 (2008).

[2] H. Song and U. W. Heinz, Phys. Lett. B 658, 279 (2008); Phys. Rev. C 78, 024902 (2008).

[3] Z. Xu, C. Greiner, and H. Stocker, Phys. Rev. Lett. 101, 082302 (2008); Z. Xu and C. Greiner, Phys. Rev. C 79, 014904 (2009).

[4] G. Ferini, M. Colonna, M. Di Toro, and V. Greco, Phys. Lett. B 670, 325 (2009); V. Greco, M. Colonna, M. Di Toro, and G. Ferini, Prog. Part. Nucl. Phys. 62, 562 (2009).

[5] A. Adare et al. PHENIX Collaboration, Phys. Rev. Lett. 98, 162301 (2007); S. S. Adler et al. PHENIX Collaboration, ibid. 91, 182301 (2003).

[6] J. Adams et al. STAR Collaboration, Phys. Rev. C 72, 014904 (2005).

[7] B. B. Back et al. PHOBOS Collaboration, Phys. Rev. C 72 , 051901(R) (2005).

[8] L. P. Csernai, J. I. Kapusta, and L. D. McLerran, Phys. Rev. Lett. 97, 152303 (2006); J. I. Kapusta, J. Phys. G: Nucl. Part. Phys. 34, S295 (2007).

[9] P. Chakraborty and J. I. Kapusta, Phys. Rev. C 83, 014906 (2011).

[10] T. Hirano, Nucl. Phys. A 769, 71 (2006).

[11] P. Zhuang, J. Hufner, S. P. Klevansky, and L. Neise, Phys. Rev. D 51, 3728 (1995); P. Rehberg, S. P. Klevansky, and J. Hufner, Nucl. Phys. A 608, 356 (1996).

[12] J. W. Chen, M. Huang, Y. H. Li, E. Nakano, and D. L. Yang, Phys. Lett. B 670, 18 (2008); J. W. Chen, C. T. Hsieh, and H. H. Lin, ibid. 701, 327 (2011).

[13] P. K. Kovtun, D. T. Son, and A. O. Starinets, Phys. Rev. Lett. 94, 111601 (2005).
[14] H. Niemi, G. S. Denicol, P. Huovinen, E. Molnar, and D. H. Rischke, Phys. Rev. Lett. 106, 212302 (2011).

[15] C. Shen and U. W. Heinz, Phys. Rev. C 83, 044909 (2011); H. Song and U. W. Heinz, ibid. 81, 024905 (2010).

[16] J. R. Bhatt, H. Mishra, and V. Sreekanth, J. High Energy Phys. 11 (2010) 106.

[17] J. Peralta-Ramos and G. Krein, Int. J. Mod. Phys. Conf. Ser. 18, 204 (2012); Phys. Rev. C 84, 044904 (2011).

[18] A. Wiranata, V. Koch, M. Prakash, and X. N. Wang, Phys. Rev. C 88, 044917 (2013); A. Wiranata and M. Prakash, ibid. 85, 054908 (2012).

[19] J. Noronha-Hostler, J. Noronha, and C. Greiner, Phys. Rev. C 86, 024913 (2012); Phys. Rev. Lett. 103, 172302 (2009).

[20] A. S. Khvorostukhin, V. D. Toneev, and D. N. Voskresensky, Phys. Atom. Nucl. 74, 650 (2011); Nucl. Phys. A 845, 106 (2010); 915, 158 (2013).

[21] M. Buballa, K. Heckmann, and J. Wambach, Prog. Part. Nucl. Phys. 67, 348 (2012).

[22] A. Dobado and S. N. Santalla, Phys. Rev. D 65, 096011 (2002); A. Dobado and F. J. Llanes-Estrada, ibid. 69, 116004 (2004).

[23] J. W. Chen, Y. H. Li, Y. F. Liu, and E. Nakano, Phys. Rev. D 76, 114011 (2007); E. Nakano, arXiv:hep-ph/0612255.

[24] K. Itakura, O. Morimatsu, and H. Otomo, Phys. Rev. D 77, 014014 (2008).

[25] A. Muronga, Phys. Rev. C 69, 044901 (2004).

[26] D. Fernandez-Fraile and A. Gomez Nicola, Eur. Phys. J. C 62 , 37 (2009); Eur. Phys. J. A 31, 848 (2007); Int. J. Mod. Phys. E 16, 3010 (2007).

[27] R. Lang, N. Kaiser, and W. Weise, Eur. Phys. J. A 48, 109 (2012); R. Lang and W. Weise, ibid. 50, 63 (2014).

[28] S. Mitra, S. Ghosh, and S. Sarkar, Phys. Rev. C 85, 064917 (2012) 
[29] S. Pal, Phys. Lett. B 684, 211 (2010).

[30] M. I. Gorenstein, M. Hauer, and O. N. Moroz, Phys. Rev. C 77, 024911 (2008).

[31] G. S. Denicol, C. Gale, S. Jeon, and J. Noronha, Phys. Rev. C 88, 064901 (2013).

[32] N. Demir and S. A. Bass, Phys. Rev. Lett. 102, 172302 (2009).

[33] D. Q. Fang, Y. G. Ma, and C. L. Zhou, Phys. Rev. C 89, 047601 (2014).

[34] N. Sadooghi and F. Taghinavaz, Phys. Rev. D 89, 125005 (2014).

[35] S. Gavin, Nucl. Phys. A 435, 826 (1985).

[36] M. Prakash, M. Prakash, R. Venugopalan, and G. Welke, Phys. Rep. 227, 321 (1993).

[37] J. L. Anderson and H. R. Witting, Physica 74, 466 (1973); 74, 489 (1973).

[38] P. Danielewicz, Phys. Lett. B 146, 168 (1984).

[39] R. Hakim, L. Mornas, P. Peter, and H. D. Sivak, Phys. Rev. D 46, 4603 (1992); R. Hakim and L. Mornas, Phys. Rev. C 47, 2846 (1993).

[40] D. N. Zubarev, Non-equilibrium Statistical Thermodynamics (New York, Consultants Bureau, 1974).
[41] R. Kubo, J. Phys. Soc. Jpn. 12, 570 (1957).

[42] S. Ghosh, Int. J. Mod. Phys. A 29, 1450054 (2014).

[43] A. J. Niemi and G. W. Semenoff, Ann. Phys. (NY) 152, 105 (1984).

[44] S. Mallik and S. Sarkar, Eur. Phys. J. C 61, 489 (2009).

[45] A. Hosoya, M. Sakagami, and M. Takao, Ann. Phys. (NY) 154, 229 (1984).

[46] M. Post, S. Leupold, and U. Mosel, Nucl. Phys. A 741, 81 (2004)

[47] S. Ghosh, S. Sarkar, and S. Mallik, Phys. Rev. C 82, 045202 (2010).

[48] S. Ghosh and S. Sarkar, Nucl. Phys. A 870, 94 (2011).

[49] S. Ghosh and S. Sarkar, Eur. Phys. J. A 49, 97 (2013).

[50] J. Beringer et al. (Particle Data Group), Phys. Rev. D 86, 010001 (2012).

[51] H. A. Weldon, Phys. Rev. D 28, 2007 (1983).

[52] S. Ghosh, G. Krein, and S. Sarkar, Phys. Rev. C 89, 045201 (2014)

[53] E. H. Kennard, Kinetic Theory of Gases, with an Introduction to Statistical Mechanics (McGraw-Hill, New York, 1938). 